\title{
Variational principle for time-periodic quantum systems
}

\author{
Nils Krüger \\ Carl von Ossietzky Universität, Institut für Physik, D-26111 Oldenburg, Germany
}

(Dated: July 27, 2020)

\begin{abstract}
A variational principle enabling one to compute individual Floquet states of a periodically timedependent quantum system is formulated, and successfully tested against the benchmark system provided by the analytically solvable model of a linearly driven harmonic oscillator. The principle is particularly well suited for tracing individual Floquet states through parameter space, and may allow one to obtain Floquet states even for very high-dimensional systems which cannot be treated by the known standard numerical methods.
\end{abstract}

Keywords: Variational principle, periodically driven quantum systems, Floquet states, quasienergy

\section{INTRODUCTION}

The Rayleigh-Ritz variational principle has proven to be of outstanding practical value for the approximate determination of a quantum system's ground state. For any trial state $|\psi\rangle$ one has the inequality

$$
R[|\psi\rangle] \equiv \frac{\langle\psi|H| \psi\rangle}{\langle\psi \mid \psi\rangle} \geq E_{0}
$$

where $H$ denotes the system's Hamiltonian, assumed to be time-independent, and $E_{0}$ is its ground-state energy. Hence, inserting an appropriate ansatz for the ground state, which should embody its key features on the one hand, and depend on convenient variational parameters on the other, one obtains an upper bound on $E_{0}$; minimizing $R$ with respect to the parameters, this bound often is found to be fairly tight. As has been concluded by Griffiths, "the variational principle is extaordinarily powerful, and embarrassingly easy to use" [1].

The purpose of the present paper is to point out that there also is a variational principle which enables one to compute Floquet states of a periodically timedependent quantum system. Such Floquet states have met with considerable interest recently; among others, they have been invoked for investigating the dynamics of atomic quantum gases in periodically driven optical lattices 2, and they lead to a natural explanation of the spontaneous breaking of time-translation symmetry occurring in so-called Floquet time cystals [3-6]. Since their quasienergies constitute infinite, ladder-like classes of equally spaced representatives, the Floquet states do generally not possess a natural order; in particular, in most cases there is no "Floquet ground state". Thus, the idea of the Rayleigh-Ritz principle (1) cannot be transferred one-by-one to Floquet systems. Nonetheless, it will be shown that there exists a similar variational principle which may allow one to compute Floquet states even for systems which are so large that they are no longer amenable to any other technique available so far.

The paper is organized as follows: For the convenience of the reader, the salient features of the Floquet picture are summarized in the subsequent Sec. III The new variational principle for Floquet states then is formulated in Sec. III and tested numerically with the help of an ana- lytically solvable model system in Sec. IV] An outlook towards future applications of the principle is given in the concluding Sec. V

\section{THE FLOQUET CONCEPT}

Consider a quantum system governed by a Hamiltonian which depends periodically on time $t$ with period $T$,

$$
H(t)=H(t+T),
$$

acting on the system's Hilbert space $\mathcal{H}$. The Floquet theorem asserts that the associated time-evolution operator $U(t, 0)$, mapping any initial state $|\psi(0)\rangle$ to the state $|\psi(t)\rangle$ which evolves from the former after time $t$,

$$
|\psi(t)\rangle=U(t, 0)|\psi(0)\rangle
$$

can be factorized to read $7-10$.

$$
U(t, 0)=P(t) \exp (-\mathrm{i} G t / \hbar),
$$

where $P(t)=P(t+T)$ is periodic in time and unitary, while the time-independent operator $G$ is self-adjoint. With $P(0)=P(T)=\mathbb{1}$, the one-cycle evolution operator then takes the form

$$
U(T, 0)=\exp (-\mathrm{i} G T / \hbar) .
$$

Generally, the spectral problem posed by $U(T, 0)$ on $\mathcal{H}$ may be quite involved [11 14; here we simply assume that $U(T, 0)$ possesses a pure point spectrum of eigenvalues $\exp \left(-\mathrm{i} \varepsilon_{n} T / \hbar\right)$ accompanied by normalized eigenstates $|n\rangle$,

$$
U(T, 0)|n\rangle=\exp \left(-\mathrm{i} \varepsilon_{n} T / \hbar\right)|n\rangle .
$$

This is not a trivial proposition; of course, this is always the case if $\mathcal{H}$ is of finite dimension. The so-called quasienergies $\varepsilon_{n}$ then constitute the eigenvalues of $G$. Due to the multi-valuedness of the complex logarithm they are defined only up to an integer multiple of $\hbar \omega$, where $\omega=2 \pi / T$ is the angular frequency implied by the period $T$.

The "stroboscopic" eigenvalue problem (6) already leads to one of the decisive benefits of the Floquet picture. 
Expanding an initial state with respect to the eigenstates $|n\rangle$ of $U(T, 0)$,

$$
|\psi(0)\rangle=\sum_{n}|n\rangle\langle n \mid \psi(0)\rangle,
$$

the combination of Eqs. (3) and (4) gives, for any time $t$,

$$
\begin{aligned}
|\psi(t)\rangle & =\sum_{n}\langle n \mid \psi(0)\rangle P(t) \exp (-\mathrm{i} G t / \hbar)|n\rangle \\
& =\sum_{n}\langle n \mid \psi(0)\rangle\left|u_{n}(t)\right\rangle \exp \left(-\mathrm{i} \varepsilon_{n} t / \hbar\right),
\end{aligned}
$$

where the Floquet functions

$$
\left|u_{n}(t)\right\rangle=P(t)|n\rangle
$$

inherit the $T$-periodicity of $H(t)$ and $P(t)$, so that

$$
\left|u_{n}(t)\right\rangle=\left|u_{n}(t+T)\right\rangle .
$$

Thus, when expanded with respect to the Floquet states

$$
\left|\psi_{n}(t)\right\rangle=\left|u_{n}(t)\right\rangle \exp \left(-\mathrm{i} \varepsilon_{n} t / \hbar\right),
$$

the time evolution (8) of $|\psi(t)\rangle$ proceeds with constant amplitudes $\langle n \mid \psi(0)\rangle$. In other words, the Floquet states carry time-independent occupation probabilities, despite the periodic time-dependence of their Hamiltonian, drastically simplifying the determination of the system's longtime behavior.

While the above stroboscopic approach is often found useful for numerical purposes, there also exists another, "extended" viewpoint which is particularly helpful for conceptual considerations. Inserting a Floquet state (11) into the time-dependent Schrödinger equation

$$
\mathrm{i} \hbar \frac{\mathrm{d}}{\mathrm{d} t}|\psi(t)\rangle=H(t)|\psi(t)\rangle,
$$

one immediately finds

$$
\left(H(t)+\frac{\hbar}{\mathrm{i}} \frac{\mathrm{d}}{\mathrm{d} t}\right)\left|u_{n}(t)\right\rangle=\varepsilon_{n}\left|u_{n}(t)\right\rangle .
$$

Augmented by the periodic boundary condition 10 to be satisfied by the Floquet functions, this is an eigenvalue problem which does not pose itself on the system's actual Hilbert space $\mathcal{H}$, but instead on an extended Hilbert space consisting of $T$-periodic functions [15]; this extended space is often denoted by $L_{2}[0, T] \otimes \mathcal{H}$ in the mathematical literature. Here the time $t$ is no longer regarded as an evolution parameter in the sense of Eq. (3), but rather as an additional coordinate; hence, the scalar product in this extended space is given by

$$
\langle\langle u \mid v\rangle\rangle=\frac{1}{T} \int_{0}^{T} \mathrm{~d} t\langle u(t) \mid v(t)\rangle,
$$

with $\langle\cdot \mid \cdot\rangle$ indicating the given scalar product on $\mathcal{H}$. Although it may seem strange from the viewpoint of conventional quantum physics on $\mathcal{H}$, the operator

$$
p_{t}=\frac{\hbar}{\mathrm{i}} \frac{\mathrm{d}}{\mathrm{d} t}
$$

acting on $L_{2}[0, T] \otimes \mathcal{H}$ now represents the momentum operator which is canonically conjugate to the coordinate $t$; the periodic boundary condition 10 makes sure that this operator is Hermitian. Observe that the quasienergy operator

$$
K=H(t)+p_{t}
$$

which appears on the left-hand side of Eq. (13) depends only linearly on this momentum. As a consequence, its quasienergy spectrum is unbounded both from above and from below: Assume that $\left|u_{n}(t)\right\rangle \equiv\left|u_{n, 0}(t)\right\rangle$ is a solution to the eigenvalue problem (13), so that

$$
K\left|u_{n}(t)\right\rangle=\varepsilon_{n}\left|u_{n}(t)\right\rangle .
$$

Then for any integer $m= \pm 1, \pm 2, \pm 3, \ldots$ the functions

$$
\left|u_{n, m}(t)\right\rangle=\left|u_{n}(t) \mathrm{e}^{\mathrm{i} m \omega t}\right\rangle
$$

likewise are $T$-periodic eigensolutions,

$$
K\left|u_{n, m}(t)\right\rangle=\left(\varepsilon_{n}+m \hbar \omega\right)\left|u_{n, m}(t)\right\rangle .
$$

Thus, from the perspective of the extended Hilbert space each Floquet state (11) evolving in $\mathcal{H}$ is associated with infinitely many eigensolutions of the eigenvalue problem $(13)$ in $L_{2}[0, T] \otimes \mathcal{H}$,

$$
\begin{aligned}
& \left|u_{n}(t)\right\rangle \exp \left(-\mathrm{i} \varepsilon_{n} t / \hbar\right) \\
= & \left|u_{n, m}(t)\right\rangle \exp \left[-\mathrm{i}\left(\varepsilon_{n}+m \hbar \omega\right) / \hbar\right] .
\end{aligned}
$$

Therefore, within this extended approach a quasienergy should not be regarded as a number $\varepsilon_{n}$, but rather as an infinite class of representatives spaced by $\hbar \omega$,

$$
\varepsilon_{n} \equiv\left\{\varepsilon_{n}+m \hbar \omega \mid m=0, \pm 1, \pm 2, \ldots\right\},
$$

reflecting the " $\hbar \omega$-indeterminacy" of the quasienergies which stems from taking the complex logarithm of the Floquet multipliers $\exp \left(-\mathrm{i} \varepsilon_{n} T / \hbar\right)$ encountered in the stroboscopic approach.

The observation that the quasienergy eigenvalue problem 17) plays a role which is conceptually similar to that of the stationary Schrödinger equation now allows one to transfer many notions known from timeindependent quantum mechanics to periodically timedependent quantum systems, such as the HellmannFeynman theorem, or Rayleigh-Schrödinger perturbation theory [15]. There is, however, a notable exception: The fact that each Floquet state is equipped with an infinite ladder (21) of quasienergies implies that these states cannot be ordered with respect to the magnitude of their quasienergies, and there is no "lowest" quasienergy. This means that the Rayleigh-Ritz principle (1) has no immediate counterpart in the extended Hilbert space, apparently depriving one of an efficient computational tool. In the follwing section it will be shown how this deficiency can be cured. 


\section{VARIATIONAL PRINCIPLE}

While the spectrum of the quasienergy operator (16) is unbounded from below, that of its square $K^{2}$ is nonnegative, as is the spectrum of $(K-\varepsilon)^{2}$ for any $\varepsilon$, be it an actual quasienergy eigenvalue of the system under consideration or not. Hence, one has the variational inequality

$$
F_{\varepsilon}[|\Psi\rangle] \equiv \frac{\left\langle\left\langle\Psi\left|(K-\varepsilon)^{2}\right| \Psi\right\rangle\right\rangle}{\langle\langle\Psi \mid \Psi\rangle\rangle} \geq 0,
$$

where $|\Psi\rangle \in L_{2}[0, T] \otimes \mathcal{H}$ is a suitably parametrized $T$ periodic trial function, and double angular brackets indicate the scalar product (14). Evidently, this functional $F_{\varepsilon}$ adopts its minimum value zero if and only if $|\Psi\rangle$ indeed is an eigenfunction of $K$ with quasienergy eigenvalue $\varepsilon$. Therefore, the inequality 22 can be exploited in two substantially different ways: (i) Keeping a given value of $\varepsilon$ fixed, and varying $|\Psi\rangle$, one may investigate whether there exists a Floquet function with that particular quasienergy. (ii) A potentially more powerful application of the inequality 22 emerges when $\varepsilon$ is regarded as an additional variational parameter: In that case one may "follow" an individual Floquet state in response to small changes of the system's parameters, as is exemplified in the following section.

In order to interpret the physical meaning of the numerical values adopted by the functional $F_{\varepsilon}$, consider an initial state in $\mathcal{H}$ at $t=t_{0}$,

$$
\left|\psi\left(t_{0}\right)\right\rangle=\sum_{n} a_{n}\left|u_{n}\left(t_{0}\right)\right\rangle
$$

which evolves in the course of one period $T$ into the state

$$
\left|\psi\left(t_{0}+T\right)\right\rangle=\sum_{n} a_{n}\left|u_{n}\left(t_{0}\right)\right\rangle \exp \left(-\mathrm{i} \varepsilon_{n} T / \hbar\right)
$$

Hence, for any $\varepsilon$ the absolute value of the return amplitude after one period $T$ ("raT") is given by

$$
\begin{aligned}
\operatorname{raT} & =\left|\exp (-\mathrm{i} \varepsilon T / \hbar)\left\langle\psi\left(t_{0}+T\right) \mid \psi\left(t_{0}\right)\right\rangle\right| \\
& =\left.\left|\sum_{n}\right| a_{n}\right|^{2} \exp \left(\mathrm{i}\left[\varepsilon_{n}-\varepsilon\right] T / \hbar\right) \mid \\
& \geq \sum_{n}\left|a_{n}\right|^{2} \cos \left(\left[\varepsilon_{n}-\varepsilon\right] T / \hbar\right) \\
& \geq 1-\frac{T^{2}}{2 \hbar^{2}} \sum_{n}\left|a_{n}\right|^{2}\left(\varepsilon_{n}-\varepsilon\right)^{2},
\end{aligned}
$$

having used $\cos (x) \geq 1-x^{2} / 2$, and the normalization of the state (23). On the other hand, "lifting" that state to the extended Hilbert space, thus considering

$$
|\psi(t)\rangle=\sum_{n} a_{n}\left|u_{n}(t)\right\rangle
$$

as an element of $L_{2}[0, T] \otimes \mathcal{H}$, one observes

$$
\sum_{n}\left|a_{n}\right|^{2}\left(\varepsilon_{n}-\varepsilon\right)^{2}=F_{\varepsilon}[|\psi\rangle] .
$$

With the estimate 25 holding for any $\varepsilon$, this gives

$$
\operatorname{raT} \geq 1-\min _{\varepsilon} 2 \pi^{2} \frac{F_{\varepsilon}}{(\hbar \omega)^{2}} .
$$

In particular, if $|\psi(t)\rangle$ is a Floquet function, one has $\min _{\varepsilon} F_{\varepsilon}[|\psi\rangle]=0$, giving $\mathrm{raT}=1$, as required. Hence, the value of the minimized functional $F_{\varepsilon}$ quantifies the failure of the variational solution to return to the initial state after one period, which may be taken as a measure of the "quality" of the approximate Floquet state obtained in this manner.

\section{EXAMPLE}

Let us consider a one-dimensional harmonic oscillator which is subjected to a monochromatic force with angular frequency $\omega$, as described in the position representation by the Hamiltonian

$$
H(t)=\frac{p^{2}}{2 M}+\frac{1}{2} M \omega_{0}^{2} x^{2}+\lambda x \cos (\omega t),
$$

where $M$ denotes the mass of the oscillator particle, $\omega_{0}$ is the oscillator's angular frequency, and $\lambda$ specifies the amplitude of the driving force. This is one of the few nontrivial Floquet systems which can be solved analytically [16 18, thus providing a benchmark test for the variational principle 22 .

To begin with, let us asssume that the driving frequency $\omega$ differs from the oscillator frequency $\omega_{0}$, because the quasienergy spectrum of the forced oscillator 29 becomes absolutely continuous in the resonant case $\omega=\omega_{0}[19$. The construction of the Floquet states then is based on the particular classical trajectory which shares the period $T=2 \pi / \omega$ of the driving force, that is, on the $T$-periodic solution to the classical equation of motion

$$
\ddot{\xi}=-\omega_{0}^{2} \xi-\frac{\lambda}{M} \cos (\omega t)
$$

which is

$$
\xi(t)=\frac{\lambda}{M\left(\omega^{2}-\omega_{0}^{2}\right)} \cos (\omega t) .
$$

Denoting the familiar eigenfunctions of the unforced oscillator with energy eigenvalues $E_{n}=\hbar \omega_{0}(n+1 / 2)$ by $\chi_{n}(x)$, where $n=0,1,2, \ldots$ is the usual oscillator quantum number, the desired Floquet states can be written as

$$
\begin{aligned}
\psi_{n}(x, t)= & \chi_{n}(x-\xi(t)) \exp \left(\frac{\mathrm{i}}{\hbar} M \dot{\xi}(t)(x-\xi(t)) .\right. \\
& \cdot \exp \left(-\frac{\mathrm{i}}{\hbar}\left[E_{n} t-\int_{0}^{t} \mathrm{~d} \tau L(\tau)\right]\right),
\end{aligned}
$$


where

$$
L(t)=\frac{1}{2} M \dot{\xi}^{2}(t)-\frac{1}{2} M \omega_{0}^{2} \xi^{2}(t)-\lambda \xi(t) \cos (\omega t)
$$

is the classical Lagrangian of the system, evaluated along the trajectory (31). Observing that the integral over this $T$-periodic function $L(t)$ contains a secular term which increases linearly with time, and thus contributes to the respective quasienergy, the quasienergy spectrum of the non-resonantly forced harmonic oscillator 29 ) is deduced to read

$$
\begin{aligned}
\varepsilon_{n} & =E_{n}-\frac{1}{T} \int_{0}^{T} \mathrm{~d} \tau L(\tau) \quad \bmod \hbar \omega \\
& =\hbar \omega_{0}\left(n+\frac{1}{2}\right)+\frac{\lambda^{2}}{4 M\left(\omega^{2}-\omega_{0}^{2}\right)} \bmod \hbar \omega,
\end{aligned}
$$

so that all states exhibit exactly the same ac Stark shift. This is a fairly unusual feature which reflects the integrability of the system (29). Thus, apart from a phase factor the Floquet states (32) are given by harmonic-oscillator eigenfunctions which follow the $T$-periodic oscillations of the classical trajectory (31).

In order to explore whether these Floquet states are correctly recovered by the variational principle $(22)$, one may take

$$
|\Psi\rangle=\sum_{n=\max \left(n_{0}-r, 0\right)}^{n_{0}+r} \sum_{m=-r}^{r} c_{n, m} \mathrm{e}^{\mathrm{i} m \omega t}|n\rangle
$$

as a natural general ansatz, with real coefficients $c_{n, m}$ to be used as variational parameters, and $\langle x \mid n\rangle=\chi_{n}(x)$. This ansatz now is employed for "tracing" the Floquet state which develops from the unperturbed oscillator ground state $|0\rangle$ when the driving amplitude $\lambda$ is gradually increased, while the driving frequency $\omega$ is kept fixed. The procedure is as follows: For $\lambda=0$ one has the exact solution $|\Psi\rangle=|0\rangle$ with $\varepsilon=E_{0}$, giving $F_{\varepsilon}[|\Psi\rangle]=0$. Then $\lambda$ is increased by a small amount $\delta \lambda$, and the variational state is seeded with the previous $|\Psi\rangle$. The variational state then is propagated by one step in imaginary "time", allowing the state to relax towards the ground state of $(K-\varepsilon)^{2}$, and $\varepsilon$ is updated to the value $\langle\langle K\rangle\rangle$ resulting from the propagated state; this is repeated until the value of the functional $F_{\varepsilon}$ has numerically converged to the accuracy specified. If, after convergence, the value of $F_{\varepsilon} /(\hbar \omega)^{2}$ equals zero within an acceptable tolerance, an approximate Floquet state has been found; if not, the searched Floquet state is not contained in the selected variational space.

Figure 1 displays data computed according to this procedure for the driving frequency $\omega / \omega_{0}=2 / 3$, while the parameters $n_{0}$ and $r$ specifying the variational space have been chosen as $n_{0}=0$ and $r=20$ (dotted lines) or $r=30$ (full lines), respectively. Shown here are the absolute value $\Delta \varepsilon$ of the difference between the variationally obtained $\varepsilon$ and the exact quasienergy obtained from Eq. (34) (red), together with the converged functional $F_{\varepsilon}$ (blue) vs. the driving amplitude, all scaled to

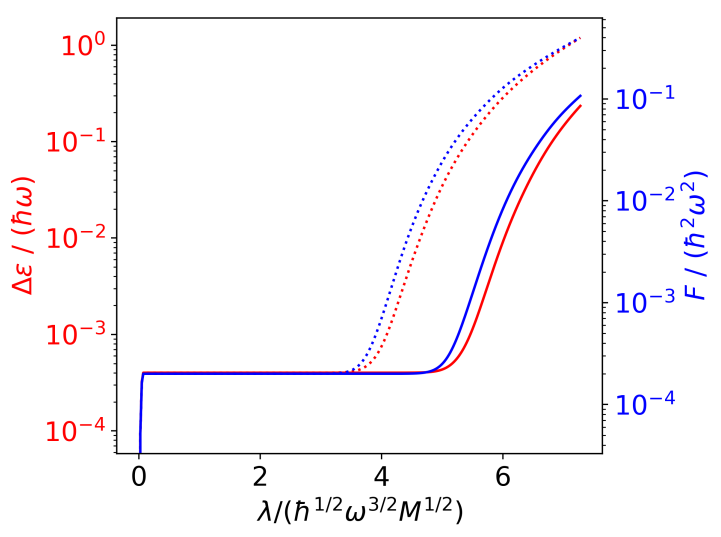

FIG. 1. Red: Difference $\Delta \varepsilon$ (absolute value) of the variationally computed quasienergy $\varepsilon_{0}$ of the Floquet state which develops continuously from the ground state $n=0$ of the unforced oscillator, and the exact quasienergy (34), vs. the scaled driving amplitude, as resulting for $\omega / \omega_{0}=2 / 3$ from the ansatz 35 with $n_{0}=0$ and $r=20$ (dotted lines) or $r=30$ (full lines). Blue: Corresponding values of the variational functional 22 after minimization.

be dimensionless. Here the stepsize $\delta \lambda / \sqrt{\hbar M \omega^{3}} \approx 0.025$ has been employed; the minimization procedure has been stopped when the absolute value of the difference between the updated value of $F_{\varepsilon} /(\hbar \omega)^{2}$ and the previous one has become lower than $\Delta=10^{-8}$. These results are extremely encouraging: Considering the data for $r=20$ first, one observes a plateau at low driving amplitudes where the variational functional adopts an almost constant small value, $F_{\varepsilon} /\left(\hbar^{2} \omega^{2}\right) \approx 2 \cdot 10^{-4}$, while $\Delta \varepsilon /(\hbar \omega)$ likewise remains small, clearly signaling that a good approximation to the exact Floquet state has been found. But then, at $\lambda / \sqrt{\hbar M \omega^{3}} \approx 4$, the variational solution suddenly becomes inacceptable. This obervation can be understood with the help of a rough estimate: According to Eq. (32), the exact Floquet state emanating from the unperturbed oscillator ground state is given by a Gaussian which sloshes along the classical trajectory (31). On the other hand, the basis states $\chi_{n}(x)$ comprising the variational space are appreciably large in the classically allowed region only, that is, between the turning points $x_{\mathrm{tp}}$ which limit the classical motion with energy $E_{n}$ in the oscillator potential, $x_{\mathrm{tp}}= \pm \sqrt{2 E_{n} /\left(M \omega_{0}^{2}\right)}$. In order to correctly represent the exact Floquet state within the variational space, the amplitude of the sloshing motion should be somewhat smaller than the largest of these turning points, implying

$$
\left|\frac{\lambda}{M\left(\omega^{2}-\omega_{0}^{2}\right)}\right| \leq \sqrt{\frac{2 E_{r}}{M \omega_{0}^{2}}}
$$

and thus providing an upper bound on the driving amplitude that can be reasonably dealt with in a variational space made up from the lowest $r+1$ oscillator functions, 


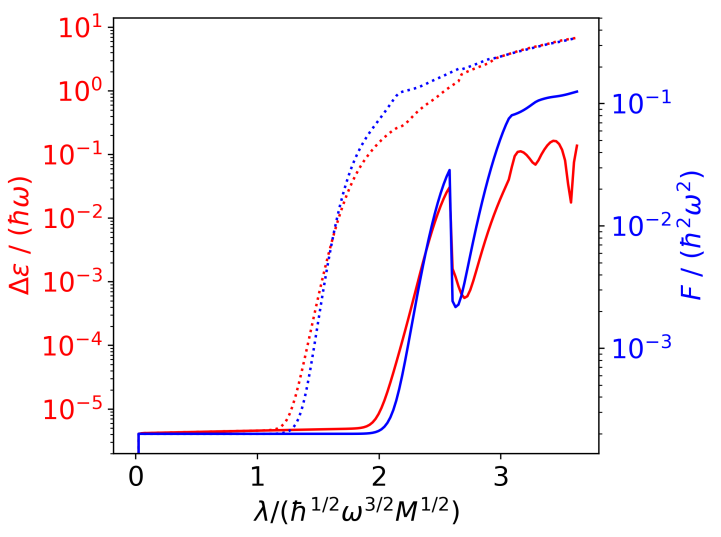

FIG. 2. As Fig. 1 for $n=n_{0}=50$, again with $r=20$ (dotted lines) and $r=30$ (full lines).

namely,

$$
\frac{\lambda^{2}}{\hbar M \omega^{3}} \leq\left[1-\left(\omega_{0} / \omega\right)^{2}\right]^{2} \frac{\omega}{\omega_{0}}(2 r+1)
$$

Inserting $\omega / \omega_{0}=2 / 3$, and $r=20$, one finds $\lambda / \sqrt{\hbar M \omega^{3}} \leq$ 6.5 , which is in acceptable agreement with the behavior shown by the dotted lines in Fig. 1, keeping in mind that the actual "critical" driving amplitude should be somewhat smaller than the order-of-magnitude estimate (37).

Thus, when the variational space is enlarged, larger driving amplitudes become admissible. For instance, when $r$ is increased to $r=30$, while $\omega / \omega_{0}$ is kept fixed, the estimate (37) gives $\lambda / \sqrt{\hbar M \omega^{3}} \leq 8.0$. That is, the range of manageable driving amplitudes is increased by a factor of 1.2 in comparson with the previous calculation, in fair agreement with the numerical data displayed in Fig 1 .

The numerical strategy suggested in this work for following an individual Floquet state through parameter space is not restricted to the Floquet state emanating from the ground state of the system in the absence of the drive, but applies the any state. In order to substantiate this claim, Fig. 2 depicts analogous numerical data obtained when tracing the Floquet state originating from the unperturbed oscillator state $n=50$, again for $\omega / \omega_{0}=2 / 3$, computed in variational spaces spanned by the ansatz states (35) with $n_{0}=50$ and $r=20$ (dotted lines) or $r=30$ (full lines). Once again, the results speak for themselves: The driving amplitudes above which the exact Floquet state is no longer adequately represented by the respective variational ansatz are well discernible; below these amplitues the variational principle 22 provides excellent approximations.

\section{CONCLUSION}

The customary computational strategies for determining the Floquet states of a periodically time-dependent quantum system rely either on Eq. (6), requiring the computation and diagonalization of the system's one-cycle evolution operator $U(T, 0)$, or on the "extended" eigenvalue problem (13), enforcing the use of a sufficiently large basis of $L_{2}[0, T] \otimes \mathcal{H}$. While this may be no problem when dealing with periodically driven single-particle systems, say, it soon becomes impractical when investigating periodically driven many-body systems. For such high-dimensional systems the variational principle 22 . may unfold its full power, enabling one to compute individual Floquet states even for systems so large that the determination of the full quasienergy spectrum would be neither feasible nor even desirable.

The particular model system that has been employed here for testing the new variational principle, the linearly driven harmonic oscillator, certainly is not typical from the Floquet point of view: Being explicitly integrable, its quasienergies (34) and Floquet states (32) can be labeled by the quantum number $n$ of the harmonicoscillator state to which they are continuously connected when the driving amplitude goes to zero. This is no longer the case for more generic systems, such as periodically forced anharmonic oscillators which possess a classial conterpart exhibiting chaotic dynamics. In such systems one encounters a quasienergy spectrum with a dense net of anticrossings [10, 20, thwarting the notion of continuity. Nonetheless, it is surmised that the idea of "tracing" an individual Floquet state through parameter space will also work for such more realistic systems, helping one to identify those Floquet states which are most important for understanding a given system's experimentally observable properties.

Finally, it needs to be stressed that the far-reaching progress made recently in the areas of machine learning and hardware design will allow one to solve variational problems even with very large numbers of variational parameters in the near future. Therefore, it is anticipated that the combined use of the variational principle (22), modern hardware, and intelligent algorithms will enable one to investigate truly large Floquet systems which are way beyond the realm of the previously used standard numerical methods.

\section{ACKNOWLEDGMENTS}

This work has been supported by the Deutsche Forschungsgemeinschaft (DFG, German Research Foundation) through Project No. 397122187. The author wishes to thank the members of the Research Unit FOR 2692 for many stimulating discussions. 
[1] D. J. Griffiths, Introduction to Quantum Mechanics, Second Edition (Pearson Prentice Hall, Upple Saddle River, 2005).

[2] A. Eckardt, Colloquium: Atomic quantum gases in periodically driven optical lattices, Rev. Mod. Phys. 89, 011004 (2017).

[3] M. Holthaus and M. E. Flatté, Subharmonic generation in quantum systems, Phys. Lett. A 187, 151 (1994).

[4] D. V. Else, B. Bauer, and C. Nayak, Floquet time crystals, Phys. Rev. Lett. 117, 090402 (2016).

[5] C. W. von Keyserlingk, V. Khemani, and S. L Sondhi, Absolute stability and spatiotemporal long-range order in Floquet systems, Phys. Rev. B 94, 085112 (2016).

[6] N. Y. Yao, A. C. Potter, I.-D. Potirniche, and A. Vishwanath, Discrete time crystals: rigidity, criticality, and realizations, Phys. Rev. Lett. 118, 030401 (2017).

[7] W. R. Salzman, Quantum mechanics of systems periodic in time, Phys. Rev. A 10, 461 (1974).

[8] S. R. Barone, M. A. Narcowich, and F. J. Narcowich, Floquet theory and applications, Phys. Rev. A 15, 1109 (1977).

[9] F. Gesztesy and H. Mitter, A note on quasi-periodic states, J. Phys. A: Math. Gen. 14, L79 (1981).

[10] M. Holthaus, Tutorial: Floquet engineering with quasienergy bands of periodically driven optical lattices, J. Phys. B: At. Mol. Opt. Phys. 49, 013001 (2016).

[11] J. S. Howland, Floquet operators with singular spectrum. I, Ann. Inst. H. Poincaré 50, 309 (1989).
[12] J. S. Howland, Floquet operators with singular spectrum. II, Ann. Inst. H. Poincaré 50, 325 (1989).

[13] J. S. Howland, Floquet operators with singular spectrum. III, Ann. Inst. Henri Poincaré 69, 265 (1998).

[14] J. S. Howland, Quantum Stability. In: "Schrödinger Operators: The Quantum Mechanical Many Body Problem." Lecture Notes in Physics 403, 100 (SpringerVerlag, Berlin Heidelberg, 1992).

[15] H. Sambe, Steady States and Quasienergies of a Quantum-Mechanical System in an Oscillating Field, Phys. Rev. A 7, 2203 (1973).

[16] K. Husimi, Miscellanea in Elementary Quantum Mechanics, II, Prog. Theor. Phys. 9, 381 (1953).

[17] V. S. Popov and A. M. Perelomov, Parametric excitation of a quantum oscillator. II, Sov. Phys. JETP 30, 910 (1970) [Zh. Eksp. Teor. Fiz. 57, 1684 (1969)].

[18] H. P. Breuer and M. Holthaus, Adiabatic processes in the ionization of highly excited hydrogen atoms, Z. Phys. D 11, 1 (1989).

[19] G. A. Hagedorn, M. Loss, and J. Slawny, Non-stochasticity of time-dependent quadratic Hamiltonians and the spectra of canonical transformations, J. Phys. A: Math. Gen. 19, 521 (1986).

[20] D. W. Hone, R. Ketzmerick, and W. Kohn, Statistical mechanics of Floquet systems: The pervasive problem of near degeneracies, Phys. Rev. E 79, 051129 (2009). 